\title{
Personalized Reduced 3-Lead System formation methodology for Remote Health Monitoring Applications and Reconstruction of Standard 12-Lead system
}

\section{Abstract}

Remote Health Monitoring (RHM) applications encounter limitations from technological front viz. bandwidth, storage and transmission time and the medical science front i.e. usage of 2-3 lead systems instead of the standard 12-lead (S12) system. Technological limitations constraint the number of leads to 2-3 while cardiologists accustomed with 12-Lead ECG may find these 2-3 lead systems insufficient for diagnosis. Thus, the aforementioned limitations pose self-contradicting challenges for RHM. A personalized reduced $2 / 3$ lead system is required which can offer equivalent information as contained in $\mathrm{S} 12$ system, so as to accurately reconstruct S12 system from reduced lead system for diagnosis.

In this paper, we propose a personalized reduced 3-lead (R3L) system formation methodology which employs principal component analysis, thereby, reducing redundancy and increasing SNR ratio, hence, making it suitable for wireless transmission. Accurate $\$ 12$ system is made available using personalized lead reconstruction methodology, thus addressing medical constraints. Mean $\mathrm{R}^{2}$ statistics values obtained for reconstruction of S12 system from the proposed R3L system using PhysioNet's PTB and TWA databases were $95.63 \%$ and $96.37 \%$ respectively. To substantiate the superior diagnostic quality of reconstructed leads, root mean square error (RMSE) metrics obtained upon comparing the ECG features extracted from the original and reconstructed leads, using our recently proposed Time Domain Morphology and Gradient (TDMG) algorithm, have been analyzed and discussed. The proposed system does not require any extra electrode or modification in placement positions and hence, can readily find application in computerized ECG machines.
Sidharth Maheshwari', Amit Acharyya ${ }^{1}$, Michele Schiariti², Paolo Emilio Puddu²

1 Department of Electrical Engineering, Indian Institute of Technology Hyderabad.

2 Department of Cardiovascular Sciences, Sapienza University of Rome, Italy.

Contact information:

Amit Acharyya.

$\equiv$ amit_acharyya@iith.ac.in 


\section{Introduction}

Advanced wireless technology, high speed internet facility and availability of other communication systems can be used to provide the accessibility of state-of-the-art healthcare facilities to the patients in remote and rural areas for monitoring and diagnosis of cardiovascular diseases (CVD), one of the prime causes of human mortality today. Tremendous and elaborative research regarding the prevention of CVD is being conducted by various researchers [1]. The recent advances in telemonitoring has enabled Home Monitoring [2] and has significantly enhanced the comfort-level of patients. These are, however, limited to urban areas as home monitoring would require availability of state-of-the-art health center nearby and supervision of cardiologists. Remote areas, generally, experience scarcity of both facilities and experienced practitioners, hence, remote health monitoring (RHM) can find immense application in diagnosis and treatment of large population in remote areas.

RHM, generally, involves acquisition and wireless transmission of ECG signals for diagnosis which posits technological constraints viz. bandwidth, storage and transmission time $[3,4,5]$. From the medical science perspective, cardiologists have been accustomed to standard 12-lead (S12) system due to its widespread usage since decades and S12 system being used as routine test for CVD diagnosis. The presence of multiple channels in $\$ 12$ restricts its usage in RHM applications [5], which preferably employs a reduced lead system with 2 or 3 leads. Numerous signal compression techniques are available that are employed prior wireless transmission to reduce the data size and decompress at the receiver's end to produce the originally sent signal. However, it has been reported that compression ratio and efficiency of signal compression algorithms decreases with the increase in the number of channels [6]. Hence, multichannel S12 system is discouraged for telemonitoring applications, though, advanced compression algorithms are available. The aforementioned arguments posits a requirement for an appropriate reduced lead system for RHM scenario and availability of S12 leads for better diagnosis of CVD.

We envisage a scenario where the ECG acquired in a remote area needs to be transmitted to nearby state-of-the-art health center. Remote areas lack in facilities and skilled caretakers. In such a scenario, a modification in reduced lead system used with or without standard electrode positions will not be practical. A reduced lead system with nonstandard placement of electrodes is unlikely to provide sufficient information to cardiologists. On the other hand, reduced lead systems such as EASI with 3-leads and standard electrode positions, similar to Frank Vectorcardiographic (FV) system [7], would require training of caretakers to acquire the skill of using such systems. It has also been found to contain insufficient information for diagnosis [8]. Such additional limitations from the RHM scenario can be allayed by using S12 system, as it is the clinical standard.

In this paper, we present a solution to the aforementioned limitations posed from technological and medical science front by proposing a personalized reduced 3-lead (R3L) system formation methodology. The proposed R3L system is derived from S12 system itself, thereby avoiding any extra electrode, hardware or skillset requirements. At the receivers end, we reconstruct S12 leads from proposed R3L system accurately and reliably in a personalized manner, which will help cardiologists in accurate diagnosis.

\section{Previous work and background}

\section{Existing lead systems and reconstruction methodologies}

Reconstruction of S12 system from FV system was proposed by Dower et al [9] (Dower Transform) where the transformation matrix was obtained using the human torso model assuming a homogeneous medium. It is also known as universal transformation 
matrix as it is patient independent. A population based methodology was proposed by Dawson et al [10] (Affine Transform), which was obtained using linear regression technique when applied on a set of patients. It was shown to outperform Dower transform in its accuracy to reconstruct S12 leads. Recently, a personalized methodology was proposed which has been shown to outperform both Dower and Affine transforms [11]. Though, the accuracy obtained from the personalized method is superior and sufficient for all practical purposes, the Frank's system, being a 3-lead system, cannot be used for RHM applications due to lack of dedicated acquisition system and uncomfortable electrode placement positions viz. behind the neck and posterior region.

There are several investigations on reconstruction of missing precordial leads from the remaining leads [12-15]. Three or four lead subsets e.g. I, II, $V_{2}$ or I, II, $V_{2}, V_{5}$, using patient-specific or populationspecific transformation methodologies have been evaluated. These basis leads in the aforementioned methodology is a subset of S12 system, hereafter will be referred as S12S system and hence, are readily available without any extra hardware or requirement. However, Maheshwari et al [15] have shown that such systems suffer from proximity effect and do not produce robust results compared to that obtained from Frank's leads. EASI system is a 3-lead system having electrode positions inspired from FV system, thus having standard positions, however, they have been found to contain insufficient information [8] and reconstruction of S12 from S12S has been shown to outperform reconstruction S12 from EASI.

Trobec and Tomasic [17] proposed an algorithm for selection of new lead system for wireless applications and it was shown to perform better than EASI system in reconstruction of S12 system. Finlay et al [18] proposed a new lead system using PCA for smart textiles [19] to increase the SNR and information content. The aforementioned lead systems involves multiple channel ECG acquisition and un- conventional electrode placement positions, hence, adoption of such systems would require further indepth investigation. Recently, Tsouri and Ostertag [20] have used two reduced 3-lead systems viz. I, II, $V_{2}$ (S12S) and Frank's XYZ leads, and reconstructed S12 systems by employing independent component analysis (ICA). However, as reported by the authors that the proposed methodology is adaptive, the results still deteriorate as the recording progresses and forthcoming QRS complexes are reconstructed. The authors have majorly concentrated on QRS complex which might not be sufficient in diagnosis of diseases whose biomarkers also depend upon other ECG features. ICA also encounters the algorithmic difficulty of order in which the independent components are obtained and no definite method to solve this limitation has been proposed by the authors. We think that further investigation is needed to measure the applicability of ICA in lead reconstruction.

\section{Previous works on lead reconstruction for RHM application}

None of the aforementioned methodologies aimed at finding application in remote health monitoring. In our previous works [11, 15, 21, 22], we have focused on this applications of lead reconstruction in a RHM scenario. All possible combinations of subsets of 3-leads of S12 system (S12S systems) have been used to reconstruct the missing leads using personalized methodology and have been shown to produce superior results compared to other investigations by various researchers. The S12S systems were also compared with Frank's system. It was found that the orthogonal leads of FV system produce robust and reliable results compared to S12S systems and are unaffected by proximity effect.

The major thrust till now has been on accurate and reliable lead reconstruction methodology. However, RHM applications require lead systems inclined towards signals with characteristics desired by wireless technology today i.e. high signal-to-noise 
(SNR) ratio [18], high compression ratio [6] etc. In this paper, we have proposed a personalized lead system formation methodology targeting the technological requirements of remote monitoring.

\section{Material}

In this paper, two databases have been used from PhysioNet [23]: PTBDB [24] and TWADB [25]. PTBDB is a 290 patient 15 lead database with both $\mathrm{S} 12$ and FV system simultaneously acquired and digitized at the sampling frequency of $1 \mathrm{kHz}$. First reading of all the 290 patients were used though some of the patients had extreme artifacts and paced rhythm. TWADB consists 100 records digitized at a sampling rate of $500 \mathrm{~Hz}$. TWADB contains standard 12-Lead ECG and some 2 or 3 signal recordings. Out of these only 72 patients had all the 12-leads of S12 recorded and hence, these patients were used in the study.

\section{Proposed methodology}

Fig. 1 shows summary of the proposed methodology. We envisage a scenario where Standard 12-lead ECG is being acquired in a remote or hospital-based environment. Generally, in a remote health monitoring scenario, these leads are needed to be transmitted to nearby state-of-the-art health center for diagnosis, storage and updating of patient's health record. At the transmission end, the conventional S12 acquisitions system is used to capture the ECG. Eight independent leads of the S12 system are then passed through the Lead Component (LC) ${ }^{1}$ system formation module (Section 3.2.1). Using $L C$ and $S 12$ system together the transformation coefficients are obtained by employing least-square (LS) fit method (Section 3.2.2). Then, LC leads along with the transformation coefficients can be transmitted and eventually 8 independent leads of the S12 system can be reconstructed for diagnosis at the receiver's end in an optimum way.

Figure 1: Summary of proposed methodology. The figure has been adopted from [22] and modified.
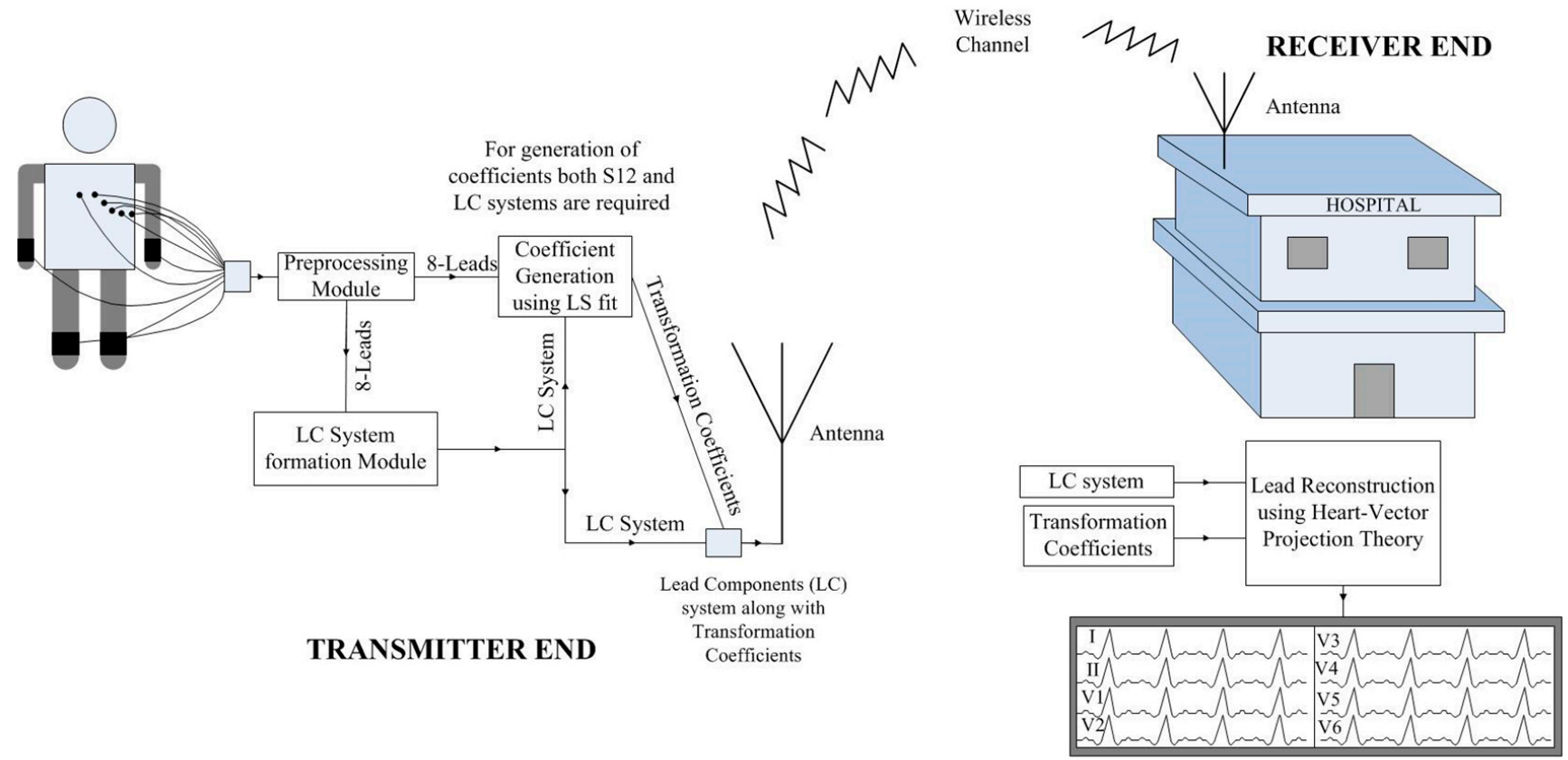

COMPUTER / MOBILE / PDA / TABLET

1. The proposed reduced 3-lead system will be referred as Lead Component (LC) system throughout the paper. 


\section{Reduced 3-Lead (R3L) system formation}

The proposed R3L has been obtained upon employing PCA on 8 independent leads of 512 system. PCA is a widely used technique for noise removal and dimensionality reduction and thus, has found applications in data compression [6], denoising [26] and reducing information redundancy [27]. It is a linear algebra technique used to find the most appropriate basis, called principal components, along which when the signal is represented has a minimum covariance. With zero co-variance, the principal components (PC) are efficient representation of the set of signals. These PCs are orthogonal to each other and each have variances associated with them. It is often found that most of the information carried by the original set of signals can be found in the first $\mathrm{k}$ principal components. The value of $k$ depends on the application.

The number of components required for our application has to be determined using Heart-Vector Projection (HVP) theory $[9,28,29]$, which states that heart can be approximated as single dipole vector, known as heart vector $\vec{H}$, fixed in 3-D space whose orientation and magnitude varies during a cardiac cycle. This dipole vector is responsible for the body surface potential observed when electrodes are placed on the body. The potential at any point on the body is the projection of $\vec{H}$ on the lead vector $(\vec{L})$ which is assumed to originate from the zeropotential region in the heart and terminates on the point of location of electrode on the body, which mathematically can be seen in equation (1).

$$
\vec{H}=X \hat{\imath}+Y \hat{\jmath}+Z \hat{k}
$$

Where $\vec{H}=X \hat{\imath}+Y \hat{\jmath}+Z \hat{k}$ and $\vec{L}=a \hat{\imath}+b \hat{\jmath}+c \hat{k}$. From this we can easily infer that all leads/channels/potential of S12 system are projection of heart vector on respective lead vectors and can be decomposed into three orthogonal components. Hence, theoretically from the standard leads it is possible to de- rive back the orthogonal components of the heart vector. We have employed PCA in this work, as it results into orthogonal components and selected first three $(k=3)$ principal components viz. $P C_{1}$, $\mathrm{PC}_{2}$ and $\mathrm{PC}_{3}$ with the intention to derive the three orthogonal heart-vector components. It should be noted that these three leads of the LC system may not correlate to FV leads [28], however, they can still be assumed to be less accurate form of heart vector components in directions different from those of Frank leads. As mentioned before, in our previous work [15] we have found FV system to produce consistent and robust reconstruction results compared to other 3-lead systems, it has motivated us to propose an orthogonal 3-lead system or the Lead Component system.

Our objective of using PCA is to reduce the inherent redundancy among the leads of S12 system. We start with the hypothesis that the first three principal components carry most of the information needed for diagnosis. The plausibility of this hypothesis is measured by reconstruction of $\mathrm{S} 12$ leads from the proposed Lead Components (LC) system and its comparison with the originally measured ECG signal. Eq. 2 shows the way in which LC system is formed for every patient. PCA is applied on the 8 independent leads of every patient and a personalized LC system is obtained. For further details on PCA interested readers may refer to [30].

$$
\left.\left[\begin{array}{c}
I \\
I I \\
V_{1} \\
V_{2} \\
V_{3} \\
V_{4} \\
V_{5} \\
V_{6}
\end{array}\right] \stackrel{P C A}{\longrightarrow}\left[\begin{array}{l}
P C_{1} \\
P C_{2} \\
P C_{3} \\
P C_{4} \\
P C_{5} \\
P C_{6} \\
P C_{7} \\
P C_{8}
\end{array}\right] \stackrel{1^{s t} \text { three } P C s}{\longrightarrow}\left[\begin{array}{l}
P C_{1} \\
P C_{2} \\
P C_{3}
\end{array}\right] \text { (LC system }\right)
$$

\section{Preprocessing module}

The preprocessing module comprises of baseline wandering (BW) removal based on discrete wavelet transform (DWT) [31] and denoising based on trans- 
lation invariant wavelet transform (TIWT) [32]. The snippet of the MATLAB code for implementation of the preprocessing module and its methodology can be found in [21].

\section{Personalized transformation coefficient generation}

The generalized form of (1) can be found in (3), where $l_{1}, l_{2}$ and $l_{3}$ are any three leads and $a, b$ and $c$ are the corresponding coefficients (lead vector components) for the electrode placed to record potential $V$.

$$
V=a l_{1}+b l_{2}+c l_{3}
$$

Using the linearity of (3), the transformation coefficients viz. $a, b$ and $c$ can be calculated using least-square fit technique, provided $V, l_{1}, l_{2}$ and $l_{3}$ are known, using eq. 4. Least square fitting is an efficient standard linear regression technique available for linear transformations, where one set of values can be transformed to another with minimum error. Such linear systems can be represented as $(A x=b)$, where the matrix $A$ is the transformation matrix. To obtain the matrix $A$, we minimize the Euclidean norm of $\|A x-b\|_{2}^{2}$ using differential equations which produces an analytical solution of $A=\left(X^{T} X\right)^{-1} X^{T} b$. Eq. (4) is the simple representation of this analytical solution for our case.

$$
\left[\begin{array}{l}
a_{i} \\
b_{i} \\
c_{i}
\end{array}\right]=\left[\begin{array}{ccc}
\sum l_{1}^{2} & \sum l_{1} \cdot l_{2} & \sum l_{1} \cdot l_{3} \\
\sum l_{1} \cdot l_{2} & \sum l_{2}^{2} & \sum l_{2} \cdot l_{3} \\
\sum l_{1} \cdot l_{3} & \sum l_{2} \cdot l_{3} & \sum l_{3}^{2}
\end{array}\right]^{-1} \times\left[\begin{array}{c}
\sum V \cdot l_{1} \\
\sum V \cdot l_{2} \\
\sum V \cdot l_{3}
\end{array}\right]
$$

For the reconstruction of $\mathrm{S} 12$ from proposed LC system, $V$ can be replaced by the $\mathrm{S} 12$ leads and $l_{1}, l_{2}$ and $l_{3}$ can be replaced by $P C_{1}, P C_{2}$ and $P C_{3}$ in (4) to obtain the corresponding transformation coefficients. In many previous works [12-15], S12S system consisting of leads $I, I I$ and $V_{2}$ have been used for reconstruction of missing precordial leads of $\$ 12$ system and it is also the most often used ba- sis lead set, hence, we compare the reconstruction results of our proposed LC system with aforementioned S12S system. To do so, V will be replaced by missing precordial leads and $l_{1}, l_{2}$ and $l_{3}$ will be replaced by $I, I I$ and $V_{2}$ in (4). The results obtained from both LC and S12S have been compared with respect to the accuracy with which they reconstruct S12 system. When (4) is employed for each patient separately, the transformation coefficients obtained are called personalized or patient-specific, while, when applied on a set of patients, they are called population-based coefficients.

A training set of 5000 samples from the middle of the recording (training set) of each patient was used in (5) for obtaining the transformation coefficients and the whole recording was used as the testing set. It should be noted that for both LC system formation, transformation coefficient generation and $\$ 12$ reconstruction only 8 independent leads of S12 system has been considered, as rest viz. III, $a V R, a V L$ and $a V F$, can be readily obtained from leads I and II and are redundant. The complete work was carried out on MATLAB (Version 7.10.0.499 R2010a).

\section{Comparison of ECG features of reconstructed and original signal}

Computerized ECG acquisition is, generally, followed by automated analysis and interpretation algorithms viz. feature extraction [33, 34], Selvester scoring $[35,36]$, fragmentation detection and identification [37, 38]. These algorithms have been found to be very sensitive and hence, requires accurate ECG to operate upon for reliable results. To verify the performance of our methodology in the context of aforementioned computerized ECG interpretation algorithms, we have employed our recently proposed Time Domain Morphology and Gradient (TDMG) [33] algorithm to extract features from PQRST complexes of both originally measured and the reconstructed signal and computed Root Mean Square Error (RMSE) (defined in next subsec- 
tion - IV E) to provide a detailed comparative study and discuss efficiency of our proposed LC system in $\mathrm{S} 12$ reconstruction.

We have used 49 patients from TWADB for this module of evaluation. 23 patients were excluded because of extreme artifacts in few or many of the leads of these patients. TDMG [33] operates accurately on a single PQRST complex, however, it is not practical to manually detect and select a PQRST complex from all the 8 leads of 49 patients for both original and reconstructed signals using both LC and S12S systems, which accounts to a total of 1568 lead annotations. To automate the process of annotation and selection of PQRST complex we used the help of two open source MATLAB files viz. nqrsdetect.m [39] and select_train.m [40]. The former function detect the fiducial points of QRS complexes and the latter helps in extraction of PQRST complexes through variations in the input arguments. To attain this complex task the results of TDMG when operated upon PQRST complexes of each patient were visually observed to fine tune the input arguments so as to obtain exactly one PQRST complex. The results obtained for the original and reconstructed signal from the TDMG algorithm were then compared using RMSE.

\section{Performance evaluation metrics}

$\mathrm{R}^{2}$ statistics, correlation coefficient $\left(r_{x}\right)$, regression coefficient $\left(b_{x}\right)[10,21,29]$ and RMSE have been used as performance evaluation metrics. All signals were mean centered before applying these metrics. $\mathrm{R}^{2}$ statistics has been used to evaluate the degree of association between the measured and the reconstructed signal. Perfect retracing of the measured wave by the reconstructed wave will be indicated by a value $100 \%$. Correlation coefficient $\left(r_{x}\right)$ [29] is a metric to estimate the similarity between two signals and regression $\left(b_{x}\right)$ [29] fairly estimates the amplitude differences between the measured and reconstructed signal. RMSE is a good measure for accuracy. The definitions of evaluation metrics are as follows 2 :

$$
\begin{gathered}
R^{2}=\left\{1-\frac{\Sigma[D(k)-O(k)]^{2}}{\Sigma[O(k)]^{2}}\right\} \times 100 \\
r_{x}=\left\{\frac{\Sigma O(k) \times D(k)}{\left(\sum O^{2}(k) \times D^{2}(k)\right)^{1 / 2}}\right\}
\end{gathered}
$$

$$
b_{x}=\left\{\frac{\sum O(k) \times D(k)}{\sum O^{2}(k) 0}\right\}
$$

$$
b_{x}=\left\{\frac{\Sigma O(k) \times D(k)}{\sum O^{2}(k) 0}\right\}
$$

In this paper, we have compared our results with three previous works of various authors $[10,12,20]$ using one or more of the aforementioned evaluation metrics. As mentioned in section IV C, we have compared our proposed reduced 3-lead system i.e. LC system with S12S system comprising of I, II and $V_{2}$ as the basis leads. For both LC and S12S systems, the same reconstruction methodology has been followed.

\section{Results}

Table 1 presents the mean $R^{2}, r_{x}$ and $b_{x}$ values for the reconstruction of $\mathrm{S} 12$ system from LC and S12S systems using both PTBDB and TWADB. The average values have been calculated over 8 independent leads of $\mathrm{S} 12$ system. The $\mathrm{R}^{2}$ values are higher for the precordial leads compared to bipolar leads $I$ and $I I$. The transformation of proposed LC system to $\$ 12$ has

2. $\mathrm{D}$ - Derived signal; $\mathrm{O}$ - Originally measured signal; $k$ - denotes $k^{\text {th }}$ sample; $\mathrm{n}$ - total number of samples 
Table 1. Mean $R^{2}, r_{x}$ and $b_{x}$ values of the transformation of LC to S12 (1) and S12S to S12 (2) systems for both TWADB (72 patients) and PTBDB (first recording of 290 patients).

\section{TWADB}

PTBDB

\begin{tabular}{l|l|l|l|l|l|l|l|l|l|l|l|l|l|l|l|l|l}
\hline I & $\|$ & $V_{1}$ & $V_{2}$ & $V_{3}$ & $V_{4}$ & $V_{5}$ & $V_{6}$ & $A V G$ & I & $\|$ & $V_{1}$ & $V_{2}$ & $V_{3}$ & $V_{4}$ & $V_{5}$ & $V_{6}$ & $A V G$ \\
\hline
\end{tabular}

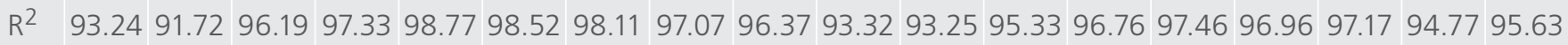

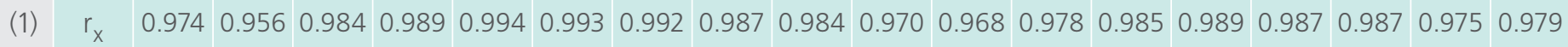

$\begin{array}{lllllllllllllllllll}b_{x} & 0.969 & 0.951 & 0.987 & 0.996 & 0.997 & 0.994 & 0.992 & 0.994 & 0.985 & 0.956 & 0.969 & 0.974 & 0.986 & 0.993 & 0.995 & 0.987 & 0.964 & 0.978\end{array}$

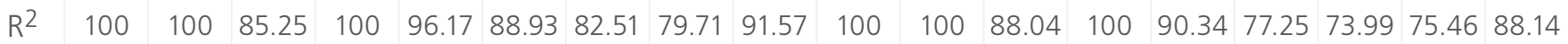

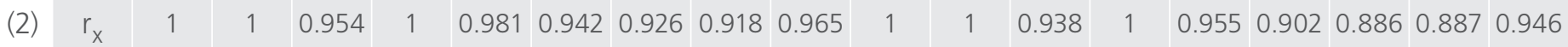

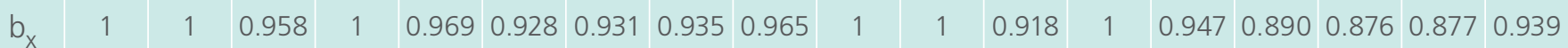

outperformed S12S system. This confirms that the information obtained from standard leads are better represented in LC system than that of a subset.
Fig. 2 presents the range of $R^{2}$ values with the mean denoted by the circle with green dot and minimum and maximum values denoted by the

Figure 2: The mean (circle with green inside) and the range (whiskers) of $R^{2}$ values for both $L C$ to $S 12$ (A-TWADB, B-PTBDB) and S12S to S12 (C-TWADB, D-PTBDB) transformations.
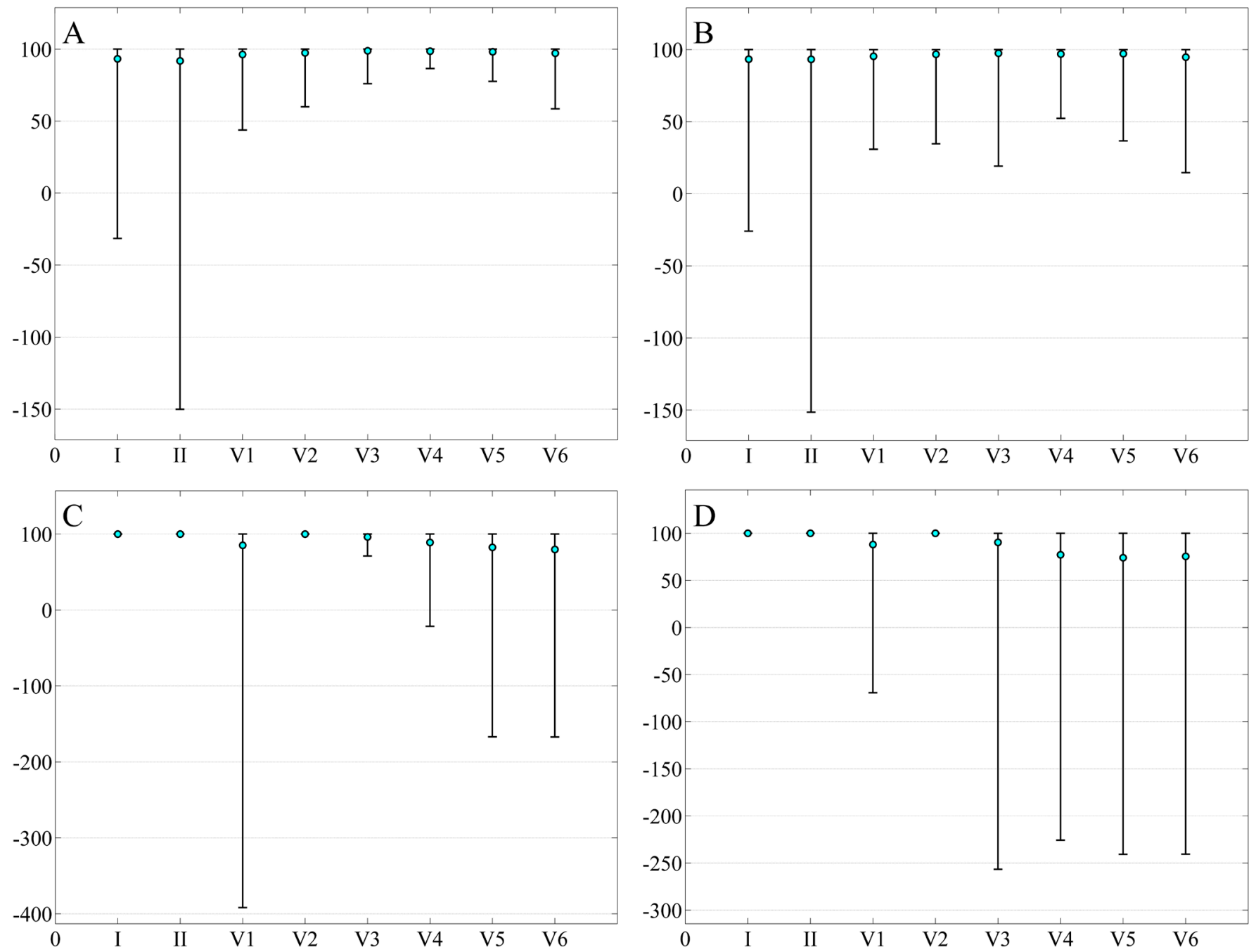
whiskers. It should be noted that Fig. 2 shows minimum $\mathrm{R}^{2}$ values being negative, which denoted that the reconstructed signal has failed to trace the original and is completely out-of-phase with it. From the range of values, it can be seen that the proposed LC system is considerably better and reliable for the reconstruction of precordial leads.

Fig. 3 shows the comparison between the originally measured (blue) and reconstructed (red) signal. One subject each from TWADB (Fig. 3 A \& B) PTBDB (Fig. 3 C \& D). The two subjects have mean
$\mathrm{R}^{2}$ values of $91.69 \%(T W A D B)$ and $88.11 \%(P T B D B)$, which is very close to the overall mean values stated in Table 1 for S12S to S12 transformation and the corresponding values for LC to S12 transformation were $96.71 \%$ and $92.64 \%$. The $\mathrm{R}^{2}$ values of respective leads have been indicated in the figure. The $\mathrm{R}^{2}$ values in Fig. 3 ranges from $54.61 \%$ to $100 \%$ and provide insight into the correspondence between the quality of reconstruction and $R^{2}$ values.

Table 2 presents the RMSE values for the features extracted from 8 independent leads of S12 system

Figure 3: Comparison between the two systems (LC and S12S) for the mean case S12S patient one each from TWADB and PTBDB. Sub-figures A and B shows comparison of 8 independent leads i.e. I, II, $V_{1}$ to $V_{6}$ for the TWADB and PTBDB and sub-figures $C$ and $D$ show a comparison of a mean case patient from PTBDB. The reconstructed lead (red) has been plotted over original lead (blue) corresponding $\mathrm{R}^{2}$ values have shown on respective plots.
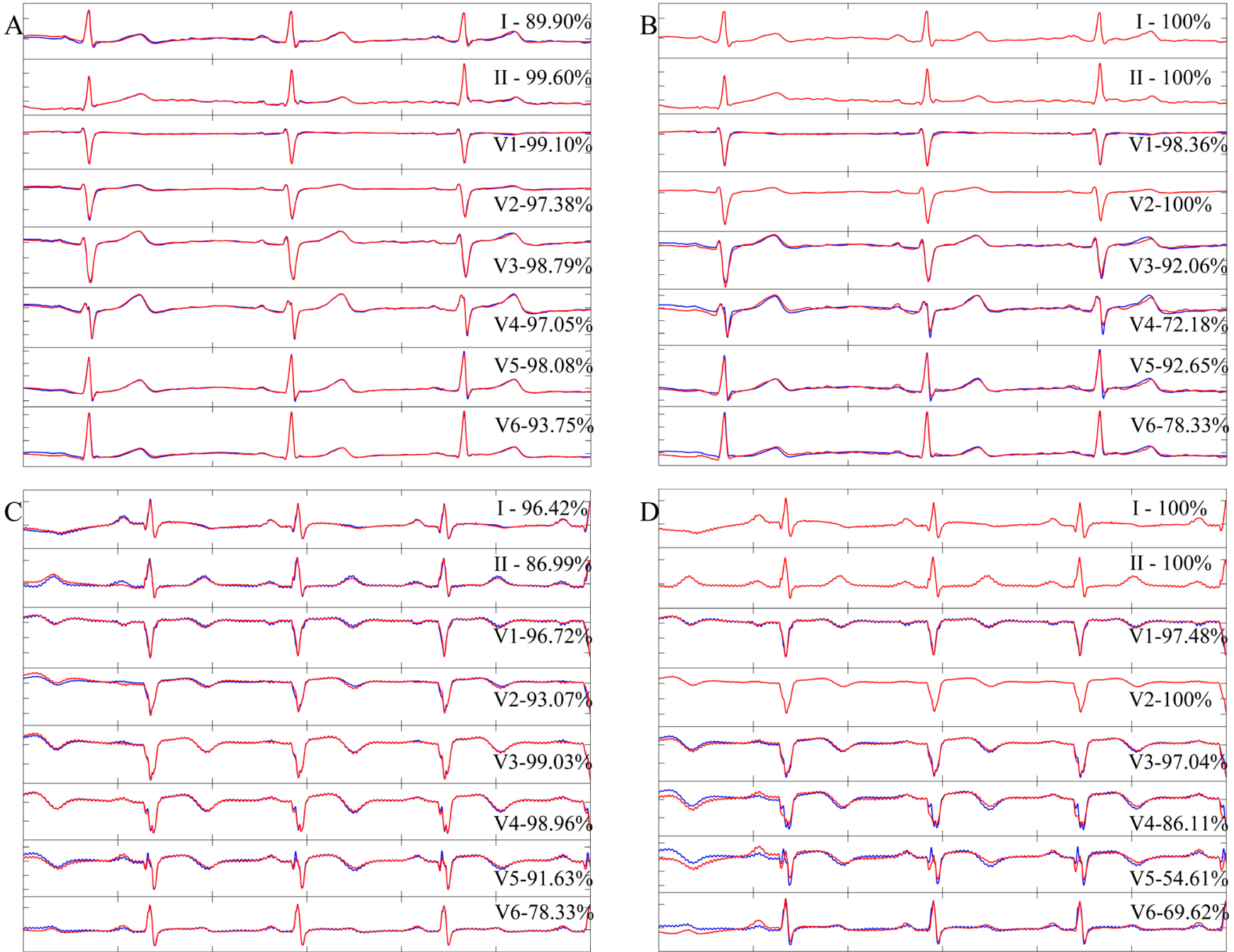
Table 2. Mean root mean square error (RMSE) values for various features extracted using TDMG for the reconstruction methodology. Mean was taken over all the 49 patients (please see section IV D). The leads $I, I I$ and $V_{2}$ formed the basis leads of S12S, so the RMSE values for them were zero and hence, has not been reported.

\begin{tabular}{|c|c|c|c|c|c|c|c|c|c|c|c|c|c|c|}
\hline \multirow[b]{2}{*}{$\mathrm{Sr}$} & \multirow[b]{2}{*}{ Feature (unit) } & \multicolumn{8}{|c|}{ LC to $\mathrm{S} 12$} & \multicolumn{5}{|c|}{$\mathrm{S} 12 \mathrm{~S}$ to $\mathrm{S} 12$} \\
\hline & & I & II & $V_{1}$ & $\mathrm{~V}_{2}$ & $V_{3}$ & $\mathrm{~V}_{4}$ & $\mathrm{~V}_{5}$ & $\mathrm{~V}_{6}$ & $V_{1}$ & $\mathrm{~V}_{3}$ & $\mathrm{~V}_{4}$ & $\mathrm{~V}_{5}$ & $\mathrm{~V}_{6}$ \\
\hline 1 & P duration (ms) & 9.755 & 13.18 & 13.43 & 10.12 & 7.306 & 9.51 & 9.632 & 7.755 & 16.86 & 12.37 & 18 & 18.82 & 19.63 \\
\hline 2 & P height $(\mu \mathrm{V})$ & 52.89 & 63.59 & 92.53 & 84.82 & 50.52 & 81.96 & 37.58 & 37.03 & 133.3 & 110.1 & 177.7 & 113.4 & 89.27 \\
\hline 3 & PR interval (ms) & 11.39 & 11.55 & 15.27 & 12.65 & 6 & 7.225 & 9.020 & 9.102 & 19.59 & 11.47 & 12.98 & 12.61 & 17.06 \\
\hline 4 & PR segment (ms) & 10.78 & 6.449 & 15.14 & 8.163 & 5.551 & 9.388 & 5.265 & 5.020 & 18.33 & 11.35 & 16.78 & 12 & 16.04 \\
\hline 5 & $\mathrm{Q}$ peak $(\mu \mathrm{V})$ & 19.58 & 14.33 & 47.73 & 60.85 & 54.27 & 52.51 & 26.20 & 16.27 & 77.22 & 111.6 & 353.4 & 62.59 & 22.27 \\
\hline 6 & QRS length (ms) & 10.65 & 7.184 & 7.633 & 3.755 & 4 & 6.816 & 5.184 & 6.122 & 9.306 & 9.143 & 11.63 & 10.61 & 10.37 \\
\hline 7 & QT interval (ms) & 16.08 & 15.8 & 26.98 & 7.551 & 18.61 & 10 & 5.469 & 8.531 & 30.61 & 21.59 & 20.33 & 11.10 & 18.65 \\
\hline 8 & $\mathrm{R}$ height $(\mu \mathrm{V})$ & 47.26 & 51.03 & 47.12 & 99.95 & 60.18 & 88.54 & 48.41 & 31.72 & 78.21 & 182.4 & 480.4 & 158.9 & 90.75 \\
\hline 9 & S peak $(\mu \mathrm{V})$ & 9.125 & 10.36 & 19.27 & 0.035 & 0.036 & 2.825 & 9.714 & 10.20 & 28.90 & 2.689 & 4.220 & 19.85 & 17.29 \\
\hline 10 & ST interval (ms) & 9.184 & 8.857 & 24.25 & 7.143 & 6.612 & 6.122 & 4.367 & 7.796 & 31.59 & 7.225 & 15.06 & 11.76 & 18.16 \\
\hline 11 & ST segment (ms) & 14.16 & 11.92 & 18.61 & 10.37 & 7.184 & 11.27 & 6.490 & 10.90 & 28.86 & 7.633 & 17.76 & 18.08 & 20.37 \\
\hline 12 & T duration (ms) & 43.32 & 64.35 & 107.5 & 114.5 & 139.0 & 105.2 & 73.27 & 43.96 & 161.2 & 199 & 202.5 & 135.7 & 98.76 \\
\hline 13 & $\mathrm{~T}$ height $(\mu \mathrm{V})$ & 22.18 & 32.95 & 55.05 & 58.62 & 71.18 & 53.84 & 37.51 & 22.51 & 82.53 & 101.9 & 103.7 & 69.50 & 50.57 \\
\hline
\end{tabular}

between the originally measured and reconstructed signal. Thirteen different features were extracted for both the methodologies ${ }^{3}$. Mean improvement of over $45 \%$ was observed in the proposed LC to S12 compared to S12S to S12 transformation.

Fig. 4 presents the box plot [41] of overall mean RMSE values of 13 different features for both the transformations LC to S12 (A) and S12S to S12 (B) The edges of the box plot are the 25th and 75th percentiles, the whiskers extend to $+/-2.7$ standard deviation ( $\sigma$ ) and rest are plotted individually.

Table 3 compares the reconstruction results presented by previous investigations to the one proposed in this paper. We have used the results obtained by Nelwan et al [12], Dawson et al [10] and Tsouri et al [20]. Nelwan el al [12] have provided median and range of correlation coefficients $\left(r_{x}\right)$ for the reconstruction of missing precordial leads from subsets of S12 system, hence, to obtain the mean value we have employed the method proposed by Hozo et al [42]. We have used the $r_{x}$ value of the basis lead set consisting leads $I, I I$ and $V_{2}$ as our proposed system is a 3-lead system. They have acquired their own private database which is publicly not available. Dawson et al [10] provided the $\mathrm{R}^{2}$ values obtained using PhysioNet's PTBDB, same as we have employed in our work. Tsouri et al [20] have also used PTBDB database and provided $r_{x}$ values in percentages. Upon comparing our results, we have found that the proposed LC to S12 transformation is superior to all three previously proposed methodologies. 
Table 3. Comparison of the proposed LC to S12 transformation with previously proposed methodologies. Mean values for 8 independent leads have been presented. Different databases were used and have been classified as follows: A - PTBDB, B - TWADB and C - self-acquired private database.

\begin{tabular}{|c|c|c|}
\hline Reconstruction Methodology & $\mathrm{R}^{2}$ values & Correlation coefficient $\left(r_{x}\right)$ \\
\hline $\begin{array}{l}\text { Proposed Lead Component (LC) system to } \\
\text { Standard 12-Lead system }\end{array}$ & $\begin{array}{l}A-95.63 \% \\
B-96.37 \%\end{array}$ & $\begin{array}{l}A-0.984(98.4 \%) \\
B-0.979(97.9 \%)\end{array}$ \\
\hline $\begin{array}{c}\text { Nelwan et al [11] - Reconstruction of missing } \\
\text { precordial leads using I, II, V2 }\end{array}$ & - & $C-0.977(97.7 \%)$ \\
\hline Dawson et al [9] - Affine Transform & $A-81.93 \%$ & - \\
\hline $\begin{array}{c}\text { Tsouri et al [19] - ICA based 12-lead ECG } \\
\text { reconstruction }\end{array}$ & - & $\begin{array}{c}\text { A - I, II, V2 - } 0.9779(97.79 \%) \\
\text { A - Frank's XYZ - } 0.9755(97.55 \%)\end{array}$ \\
\hline
\end{tabular}

Figure 4: Box plot of RMSE values. A - LC to S12 and B - S12S to S12. The labels 1-13 on the horizontal axis corresponds to the respective features extracted from TDMG as mentioned in Table 2.

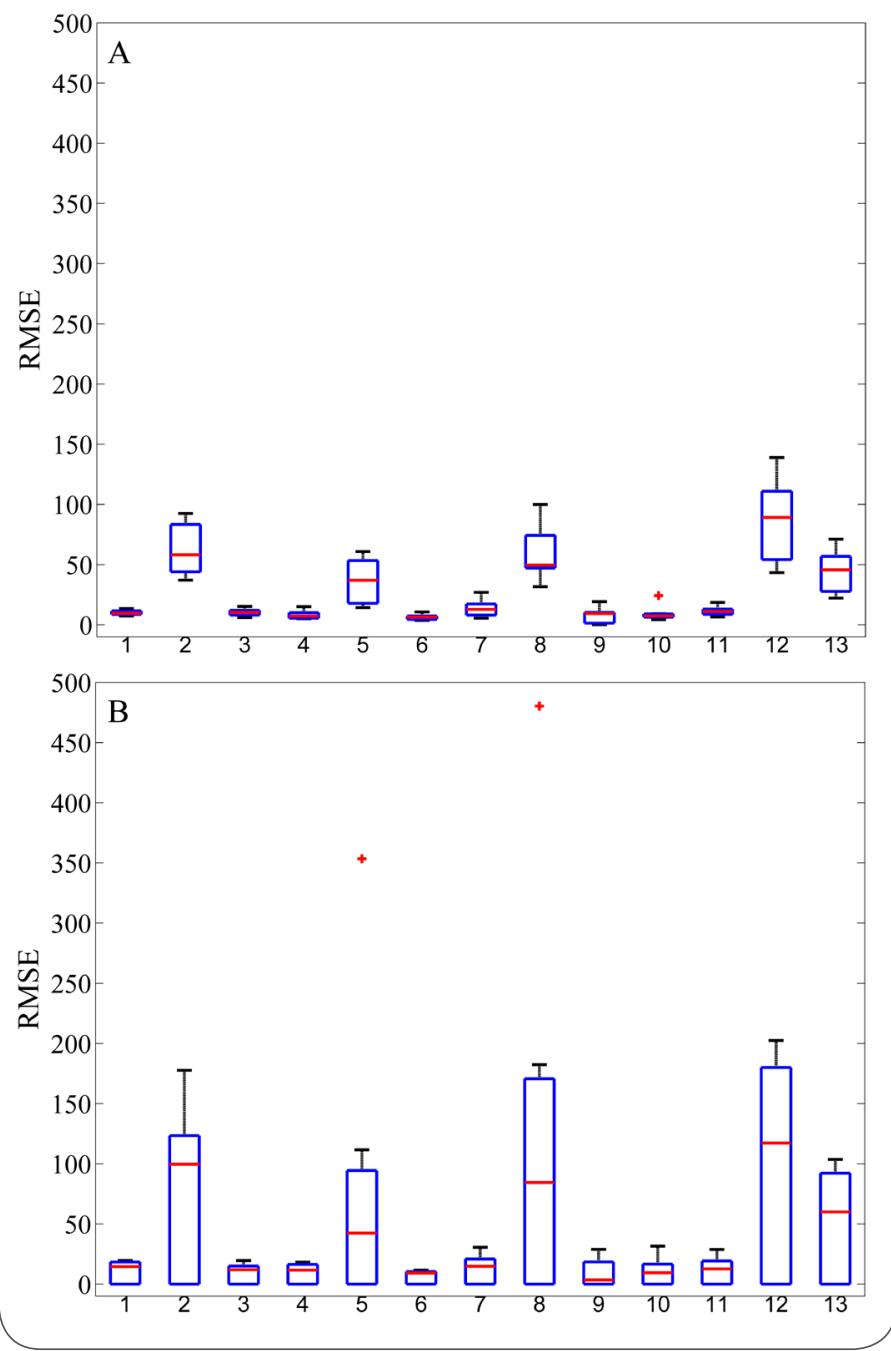

\section{Discussion}

From a wide range of $\mathrm{R}^{2}$ values in fig. 3 i.e. $54.61 \%$ to $100 \%$, we have found that $R^{2}$ value of $80 \%$ or above can be considered to have significant diagnostic value and a value of $90 \%$ or above is practically retracing the original waveform. For PTBDB and TWADB using the proposed methodology, approximately, $96 \%$ of patients were found to have mean $R^{2}$ value of $80 \%$ and above. Similarly, $91 \%$ and $93 \%$ of patients were found to have mean $R^{2}$ value of $90 \%$ and above for PTBDB and TWADB respectively. For S12S to S12 transformation, the fraction of patients above with mean $R^{2}$ values $\geq 80 \%$ were $86 \%$ (PTBDB) and $92 \%$ (TWADB). The fraction of patients with mean $\mathrm{R}^{2}$ values $\geq 90 \%$ were 76\% (PTBDB) and 83\% (TWADB). In Fig. 4, it can be seen that RMSE has higher values for peaks and nadirs e.g P height, Q peak etc as compared to the features related to time axis e.g. P duration, QRS length etc. as compared to the features related to the time axis e.g. P duration, QRS length etc. From Tables 1 and 2 and Fig. 2-4 it can be seen that the proposed reconstruction methodology has considerably outperformed the transformation of S12S to S12, especially, for leads $V_{4}$ to $V_{6}$ in terms of accuracy and robustness in $\mathrm{S} 12$ lead reconstruction which is of utmost importance for diagnosis particularly for remote healthcare scenario. The proposed methodology has yielded a mean improvement of $7.49 \%$ (PTBDB) and $4.8 \%$ 
(TWADB) over our previously proposed personalized methodologies. This improvement is significant and shows importance of selecting appropriate lead system.

In Gregg et al. [13], the authors have discussed the limitations on the re-usability of transformations coefficients. They showed that the reconstruction results obtained from the same transformation coefficients calculated from a particular measurement deteriorates when electrodes are removed and replaced for another acquisition. They attributed this deterioration mainly to the human error involved in replacement of electrodes at the same positions. Using our proposed methodology the coefficients can be recalculated every time a measurement is taken with the help of computerized ECG acquisition systems. In cases where sudden event occurs while monitoring viz. arrhythmia, then the coefficients will have to be calculated again. Computerized ECG acquisition systems can be readily used for such events. In the proposed methodology, S12 leads needs to be acquired whenever signals are needed to be transmitted and this paper mainly addresses the limitations encountered in RHM scenario. Storage of ECG data of patients in a telemonitoring system using our proposed methodology provides the following:

- Three leads require less space compared to eight leads of S12 system and hence, can be used to reduce the memory requirements.

Proposed methodology ensures accuracy and robustness in the $\$ 12$ lead reconstruction.

With the decrease in number of leads the compression ratio increases [6].

The proposed methodology does not require any change in the hardware of the, today, widely used computerized ECG acquisition system. The proposed system with the application of PCA reduces data redundancy.

Leads enriched with condensed and most important information is retained which have high Signal-to-Noise (SNR) ratios as compared to S12 leads which makes them suitable for wireless or any other mode of transmission.

The proposed system ensures the availability of S12 system using personalized lead reconstruction methodology for diagnosis.

PCA has found many applications in signal compression algorithm [6]. However, in this paper, our objective is to assimilate most of the information present in S12 leads on three leads so as to reduce the number of channels and facilitate its reconstruction. Signal compression will be the next step to be applied before transmission. ICA has been used by Tsouri and Ostertag [20], however, our PCA based methodology is comparatively superior. The major limitation of ICA is the unpredictable ordering in which ICs are obtained. In [20], ICA was applied on basis leads $I, I I$ and $V_{2}$ and Frank's XYZ leads which already have limited information, hence, ICA cannot not obtain any extra information. However, we have applied PCA on all the 8 independent leads, thereby, LC system has more information on the S12 leads. Frank's system is supposed to be an orthogonal system with independent leads as they are on different planes and ICA produces another set of orthogonal independent leads, which can be assumed to be different representation of the same information, thereby, not gaining any extra insight into the nature of S12 leads. Several other algorithms exists e.g. LDA, non-linear PCA, which can be evaluated in the context of lead reconstruction, however, this we leave upto the readers to evaluate.

\section{Conclusion}

In this paper, we proposed a personalized and robust reduced 3-lead system viz. Lead Component (LC) system, formation methodology targeting the emerging field of remote health monitoring to improve diagnosis and prognosis by overcoming the constraints from technical and medical science side. It has been shown that the proposed LC system out- 
performs the existing methodologies $[10,12,15,20$, 21] by various investigators for the reconstruction of standard 12-leads and has been verified on two widely accepted databases viz. PTBDB and TWADB of PhysioNet. The importance of an appropriate personalized reduced lead system has been showcased. We introduced PCA in this context to obtain and orthogonal 3-lead personalized system which reduces dimension of the data from 8 leads to 3 leads resulting in an increase in the SNR and Compression ratio, which favors wireless transmission of the data either to the medical practitioner's mobile or to the personalized health data repository in the cloud. As mentioned in the previous section, the personalized coefficients for reconstruction are always recalculated and retransmitted each time the contact electrodes are positioned on the patient, thus our proposed system would greatly reduce the attrition dequalification rate discussed by Gregg et al. [13].

\section{Funding}

This work was partly supported by the Department of Electronics and Information Technology (DEITY), India, under the Cyber Physical Systems Innovation Hub [grant number 13(6)/2010-CC\&BT dated 1 March 2011].

\section{Disclosure}

The acceptance of this article has been taken by the editor-in-chief of International Archives of Medicine.

\section{References}

1. Menotti A, Lanti M, Mancini A, Zanchetti A, Laurenzi M, Terradura Vagnarelli O, Puddu PE. Moderate leisure time physical activity and occurrence of cardiovascular events: a 10-year follow-up in the Gubbio Population Study. JCvD 2014;2(5):218224.

2. Bosznai I, Ender F, Santha H. Web server based remote health monitoring system. In: 32nd International Spring Seminar on Electronics Technology, 2009 (ISSE 2009), 13-17 May 2009. p. 1-6. (DOI: 10.1109/ISSE.2009.5206985)

3. Puddu PE, D'Ambrosi A, Scarparo P, Centaro E, Torromeo C, Schiariti M, et al. A Clinician's View of Next-Generation Remote Healthcare System. In: Maharatna K, Bonfiglio S, editors. Systems Design for Remote Healthcare. Springer New York, 2014. p. 1-30. (DOI: 10.1007/978-1-4614-8842-2_1)

4. Puddu PE, Morgan JM, Torromeo C, Curzen N, Schiariti M, Bonfiglio S. A Clinical Observational Study in the CHIRON Project: Rationale and Expected Results. In: Donnelly M, Paggetti C, Nugent C, Mokhtari M, editors. Impact Analysis of Solutions for Chronic Disease Prevention and Management. Springer Berlin Heidelberg, 2012, p. 74-82. (DOI: 10.1007/978-3-642-307799_10)

5. Liszka KJ, Mackin MA, Lichter MJ, York DW, Dilip P, Rosenbaum DS. Keeping a beat on the heart. Pervasive Comput. 2004 Oct; 3(4):42-9. (DOI: 10.1109/MPRV.2004.10)

6. Sharma LN, Dandapat S, Mahanta A. Multichannel ECG Data Compression Based on Multiscale Principal Component Analysis. IEEE Trans Inf Technol Biomed. 2012 July; 16(4):730-6. (DOI: 10.1109/TITB.2012.2195322)

7. Frank E. An accurate, clinically practical system for spatial vectorcardiography. Circulation. 1956; 13:737-49.

8. Hoekema R, Uijen GJH, van Oosterum A. On selecting a body surface mapping procedure. J Electrocardiol. 1999; 32(2):93101. (DOI: 10.1016/S0022-0736(99)90088-2)

9. Dower GE. A lead synthesizer for the frank system to simulate the standard 12-lead electrocardiogram. J Electrocardiol 1968; 1(1):101-16. (DOI: 10.1016/0022-0736(88)90090-8)

10. Dawson D, Yang H, Malshe M, Bukkapatnam STS, Benjamin $B$, Komanduri R. Linear affine transformations between 3-lead (Frank XYZ leads) vectorcardiogram and 12-lead electrocardiogram signals. J Electrocardiol 2009; 42(6):622-30. (DOI: 10.1016/j.jelectrocard.2009.05.007)

11. MaheshwariS, Acharyya A, Puddu PE, Mazomenos EB, Schiariti M, Maharatna K. Robust and accurate personalised reconstruction of standard 12-lead system from Frank vectordcardiographic system. Comput Methods Biomech Biomed Eng Imaging Vis. 2014. (DOI: 10.1080/21681163.2014.931029)

12. Nelwan SP, Kors JA, Meij SH, van Bemmel JH, Simoons ML. Reconstruction of the 12-lead electrocardiogram from reduced lead sets. J Electrocardiol 2004; 37(1):11-18. (DOI: 10.1016/j. jelectrocard.2003.10.004) 
13. Gregg RE, Zhou SH, Lindauer JM, Helfenbein ED, Field DQ. Limitations on the Re-use of patient specific coefficients for 12-lead ECG reconstruction. In: Computers in Cardiology 2008 Sept 14-17. p. 209-212. (DOI: 10.1109/CIC.2008.4749014)

14. Field DQ, Zhou SH, Helfenbein ED, Gregg RE, Lindauer JM. Technical challenges and future directions in lead reconstructions for reduced-lead systems. J Electrocardiol 2008; 41(6):466-73. (DOI: 10.1016/j.jelectrocard.2008.07.019)

15. Maheshwari S, Acharyya A, Puddu PE, Schiariti M. Reduced lead system selection methodology for reliable standard 12-lead reconstruction targeting personalised remote health monitoring applications. Comput Methods Biomech Biomed Eng Imaging Vis. 2014; 2(2):107-20. (DOI: 10.1080/21681163.2013.859097)

16. Finlay DD, Nugent CD, Kellet JG, Donnelly MP, McCullagh, Black ND. Synthesising the 12-lead electrocardiogram: Trends and challenges. Eur J Intern Med 2007; 18(8):566-70. (DOI: 10.1016/j.ejim.2007.04.011)

17. Trobec R, Tomasic I. Synthesis of the 12-Lead Electrocardiogram From Differential Leads. IEEE Trans Inf Technol Biomed. 2011 July; 15(4): 615-21. (DOI: 10.1109/TITB.2011.2159236)

18. Finlay DD, Nugent CD, Donnelly MP, Lux RL. Eigenleads: ECG Leads for Maximizing Information Capture and Improving SNR. IEEE Trans Inf Technol Biomed. Jan 2010; 14(1):69-78. (DOI: 10.1109/TITB.2009.2022933)

19. Axisa F, Schmitt P, Gehin C, Delhomme G, McAdams E, Dittmar A. Flexible technologies and smart clothing for citizen medicine, home healthcare, and disease prevention. IEEE Trans Inf Technol Biomed. Sept 2005; 9(3):325-336. (DOI: 10.1109/ TITB.2005.854505)

20. Tsouri GR, Ostertag MH. Patient-Specific 12-Lead ECG Reconstruction From Sparse Electrodes Using Independent Component Analysis. IEEE J Biomed Health Inform. Mar 2014; 18(2):476-82. (DOI: 10.1109/JBHI.2013.2294561)

21. Maheshwari S, Acharyya $A$, Rajalakshmi $P$, Puddu PE, Schiariti M. Accurate and reliable 3-lead to 12-lead ECG reconstruction methodology for remote health monitoring applications. In: IEEE 15th International Conference on e-Health Networking, Applications Services (Healthcom), Oct 2013. P. 233-37. (DOI: 10.1109/HealthCom.2013.6720673)

22. Maheshwari S, Acharyya A, Rajalakshmi P, Puddu PE, Schiariti M. Accurate and reliable 3-lead to 12-lead ECG reconstruction methodology for remote health monitoring applications. IRBM. Dec 2014; 35(6):341-350. (DOI: 10.1016/j.irbm.2014.07.004)

23. Goldberger AL, Amaral LAN, Glass L, Hausdorff JM, Ivanov PCh, Mark RG, et al. PhysioBank, PhysioToolkit, and PhysioNet: Components of a New Research Resource for Complex Physiologic Signals. Circulation. 2000; 101(23):e215-e220.

24. Bousseljot R, Kreiseler D, Schnabel A. Nutzung der EKG Signaldatenbank CARDIODAT der PTB ber das Internet. Biomedizinische Technik. 1995; 40(1 supp):317.
25. Moody GB. The PhysioNet / Computers in Cardiology Challenge 2008: T-Wave Alternans. In: Computers in Cardiology 2008. p. 505-8.

26. Sharma LN, Dandapat S, Mahanta A. Multiscale principal component analysis to denoise multichannel ECG signals. CIBEC 2010: 5th Cairo International Biomedical Engineering Conference; Dec 2010. p. 17-20. (DOI: 10.1109/CIBEC.2010.5716093)

27. Martis RJ, Chakraborty C, Ray AK. An Integrated ECG Feature Extraction Scheme Using PCA and Wavelet Transform. INDICON 2009: Annual IEEE India Conference, Dec 2009. p. 1-4. (DOI: 10.1109/INDCON.2009.5409439)

28. Frank E. General Theory of Heart-Vector Projection. Circulation Research. 1954; 2(3):258-70. (DOI: 10.1161/01.RES.2.3.258)

29. Levkov CL. Orthogonal electrocardiogram derived from the limb and chest electrodes of the conventional 12-lead system. Med Biol Eng Comput. 1987; 25(2):155-64. (DOI: 10.1007/ BF02442844)

30. Jolliffe IT. Principal Component Analysis. Springer Series in Statistics, Springer New York 2002.

31. Zhang D. Wavelet Approach for ECG Baseline Wander Correction and Noise Reduction. IEEE EMBS 2005: 27th Annual International Conference of the Engineering in Medicine and Biology Society, 2005. p. 1212-5. (DOI: 10.1109/IEMBS.2005.1616642)

32. Antoniadis A, Bigot J, Sapatinas T. Wavelet Estimators in Nonparametric Regression: A Comparative Simulation Study. J Stat Softw. Jun 2001; 6(6):1-83.

33. Mazomenos EB, Chen T, Acharyya A, Bhattacharya A, Rosengarten J, Maharatna K. A Time-Domain Morphology and Gradient based algorithm for ECG feature extraction. ICIT 2012: IEEE International Conference on Industrial Technology, Mar 2012. p. 117-122. (DOI: 10.1109/ICIT.2012.6209924)

34. Mazomenos EB, Biswas D, Acharyya A, Chen T, Maharatna K , Rosengarten J, et al. A Low-Complexity ECG Feature Extraction Algorithm for Mobile Healthcare Applications. IEEE J Biomed Health Inform. Mar 2013; 17(2):459-69. (DOI: 10.1109/ TITB.2012.2231312)

35. Horacek BM, Warren JW, Albano A, Palmeri MA, Rembert JC, Greenfield Jr. JC, Wagner GS. Development of an automated Selvester Scoring System for estimating the size of myocardial infarction from the electrocardiogram. J Electrocardiol. 2006; 39(2):162-8. (DOI: 10.1016/j.jelectrocard.2005.08.013)

36. Bono V, Mazomenos EB, Chen T, Rosengarten J, Acharyya A, Maharatna K, et al. Development of an Automated Updated Selvester QRS Scoring System Using SWT-Based QRS Fractionation Detection and Classification. IEEE J Biomed Health Inform. Jan 2014; 18(1):193-204. (DOI: 10.1109/ JBHI.2013.2263311) 
37. Maheshwari S, Acharyya A, Puddu PE, Schiariti M. Methodology for automated detection of fragmentation in QRS complex of Standard 12-lead ECG. IEEE EMBS 2013: 35th Annual International Conference of the IEEE Engineering in Medicine and Biology Society, 2013. p. 3789-3792. (DOI: 10.1109/ EMBC. 2013.6610369)

38. Maheshwari S, Acharyya A, Puddu PE, Mazomenos EB, Leekha G, Maharatna K, Schiariti M. An automated algorithm for online detection of fragmented QRS and identification of its various morphologies. J R Soc Interface. 2013; 10(89). (DOI: 10.1098/ rsif.2013.0761)

39. Afonso V, Tompkins WJ, Nguyen T, Luo S. ECG beat detection using filter banks. IEEE Trans Biomed Eng. 1999; 46(2):192-202. (DOI: 10.1109/10.740882)

40. Clifford GD. ECG tools. Available from: http://www.robots. ox.ac.uk/ gari/CODE/ECGtools

41. Mathworks. Boxplot. Available from: http://www.mathworks. in/help/stats/boxplot.html

42. Hozo SP, Djulbegovic B, Hozo I. Estimating the mean and variance from the median, range, and the size of a sample. BMC Med Res Methodol. Apr 2005; 5(13). (DOI: 10.1186/1471-22885-13)

\section{Comment on this article:}

\section{(f) [B] in $8+\mathbf{S}$ P}
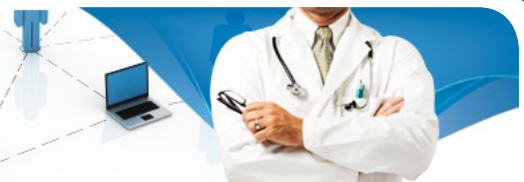

http://medicalia.org/

Where Doctors exchange clinical experiences, review their cases and share clinical knowledge. You can also access lots of medical publications for free. Join Now!

\section{Publish with iMedPub}

http://www.imed.pub

International Archives of Medicine is an open access journal publishing articles encompassing all aspects of medical science and clinical practice. IAM is considered a megajournal with independent sections on all areas of medicine. IAM is a really international journal with authors and board members from all around the world. The journal is widely indexed and classified Q1 in category Medicine. 